\title{
A Review on EEG Signals Based Emotion Recognition
}

\author{
Morteza Zangeneh Soroush'1, Keivan Maghooli', Seyed Kamaledin Setarehdan², Ali Motie Nasrabadi ${ }^{*}$ \\ 1Department of Biomedical Engineering, Science and Research Branch, Islamic Azad University, Tehran, Iran \\ ${ }^{2}$ Control and Intelligent Processing Center of Excellence, School of Electrical and Computer Engineering, College of \\ Engineering, University of Tehran, Tehran, Iran \\ ${ }^{3}$ Biomedical Engineering Department, Faculty of Engineering, Shahed University, Tehran, Iran
}

\begin{abstract}
Emotion recognition has become a very controversial issue in brain-computer interfaces ( $\mathrm{BCls})$. Moreover, numerous studies have been conducted in order to recognize emotions. Also, there are several important definitions and theories about human emotions. In this paper we try to cover important topics related to the field of emotion recognition. We review several studies which are based on analyzing electroencephalogram (EEG) signals as a biological marker in emotion changes. Considering low cost, good time and spatial resolution, EEG has become very common and is widely used in most $\mathrm{BCl}$ applications and studies. First, we state some theories and basic definitions related to emotions. Then some important steps of an emotion recognition system like different kinds of biologic measurements (EEG, electrocardiogram [EEG], respiration rate, etc), offline vs online recognition methods, emotion stimulation types and common emotion models are described. Finally, the recent and most important studies are reviewed.

Keywords: Emotion, affect; Recognition; Classification; Electroencephalogram
\end{abstract}

*Correspondence to

Keivan Maghooli, Department of Biomedical Engineering, Science and Research Branch, Islamic Azad University, Tehran, Iran

Tel: +98(912)2993212;

Email:k_maghooli@srbiau.ac.ir

Published online October 8, 2017

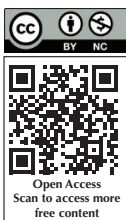

Citation: Zangeneh Soroush M, Maghooli K, Kamaledin Setarehdan S, Motie Nasrabadi A. A review on EEG signals based emotion recognition. Int Clin Neurosci J. 2017;4(4):118-129. doi:10.15171/icnj.2017.01.

\section{Introduction}

Everyone knows original and basic emotions such as happiness, fear, anger, disgust, sadness and surprise. But neuroscientists and researchers have no consensus about the nature of emotions. There are 2 opinions about emotions: one approach considers emotions as general states of individuals and the other one knows emotions as physiological interactions. ${ }^{1}$ Imagine a person driving a car while another car approaches and causes him to deviate from the road. At first that individual probably experiences fear and anger. According to the first view, fear comes from the inference that one might be in anger and that anger is because of the driver who has just put him in danger. Thagard, ${ }^{1}$ Oately $^{2}$ and Nussbaum ${ }^{3}$ believe in the first approach. Oately demonstrated how original emotions have a strong relation with executing goals. In other words, people become happy while approaching their goals and sad when they fail. People become frightened when they experience trouble or feel threatened. Therefore, we can consider emotions a general representation of our problems. ${ }^{1}$ In contrast to the first view, the second approach emphasis on physical and physiological interactions. When someone causes an individual driving a car to deviate off the road, their heart rate, blood pressure and respiration rate increase. Feelings (like fear or anger, etc) originate from the brain's responses to these physiological changes and not from the interpretation of the situation. James introduced this approach for the first time in 1884. Psychologically speaking, in terms of emotion classification there are 2 basic theories: Plutchik's theory and Ekman's theory. The first theory classifies emotions into 2 different categories: basic emotions and secondary ones. These emotions are as follows: anticipation, joy, trust, sadness, fear, surprise, anger, disgust. Secondary emotions come from a combination of these elementary feelings. These emotions are as follows: love, optimism, aggressiveness, submission, contempt, awe, remorse and disapproval. Ekman's theory is known as a discrete model. He introduced six basic emotions: fear, sadness, happiness, surprise, disgust, anger. ${ }^{4}$ After that, the number of these emotions increased to 15. James and Lange in the 19th century introduced another theory. In this theory environmental variations cause physiological changes in our autonomous nervous system and consequently cause different emotions. On the other hand, physiological changes cause emotions. Therefore, researchers analyze signals and images related to these physiological changes in order to recognize feelings and classify emotions. However, physiological signals introduce some problems like noise, artifacts, etc. Another problem is that we cannot visually recognize emotions from physiological

(C) 2017 The Author(s). This is an open-access article distributed under the terms of the Creative Commons Attribution-NonCommercial 4.0 International License (http://creativecommons.org/licenses/by-nc/4.0/) which permits copy and redistribute the material just in noncommercial usages, provided the original work is properly cited. 
signals and need computerized processes. ${ }^{5-11}$ Also, there are other factors which affect emotions, such as sex, age and race. Usually, researchers consider these parameters while studying emotions. Besides the discrete model of emotions, there is another model which Lang proposed and called valence-arousal model. In this model, valence and arousal values are assigned to each emotion. In other words, in this model emotions are a continuous spectrum of valence and arousal values and generally emotions are plotted in a $2 \mathrm{D}$ coordination called valence-arousal plane.

There are 4 important steps in emotion recognition systems: physiologic records, emotion stimulation, online or offline recognition and stimulated emotions and emotion models.

\section{Physiologic Records}

Emotion status is reflected by physiological changes, which is why biological signals and images are recorded in order to recognize emotions. Some biological systems in the human body and their indexes are described as follows:

1-Cardiovascular system: electrocardiogram (ECG), heart rate variability (HRV), cardiac output, blood pressure, etc. 2- Respiratory system: respiration rate, etc.

3- Muscular system: electromyogram (EMG) signals, etc. 4- Brain activity: EEG signals, etc.

Figure 1 shows some types of these signals.

EEG signals due to their simplicity to analyze and good time and spatial resolution have become common and useful in most BCI applications such as emotion recognition. Also, EEG recording systems are cheap and accessible. Previous studies show that by recording and processing EEG signals we can achieve very good results in terms of emotion classification. So a decision was made to explain and review some previous studies related to emotion classification through EEG signals.

\section{Emotion Stimulation}

The way emotions are evoked plays an important role in emotion recognition systems. Some believe that video clips can stimulate human emotions the best while others find music or memories the most effective way. What is clear is that the stronger the stimulation is the richer the database will be. By using good and strong stimulation, emotion recognition is more likely to be performed with better results and higher accuracy. There are some types of stimulation as follow: pictures, ${ }^{12-43}$ video clips, ${ }^{44-60}$ music, ${ }^{61-76}$ memories, ${ }^{77}$ self-induction, ${ }^{78,80,81}$ environment elicitation like light, humidity and temperature, ${ }^{79}$ games, ${ }^{82}$ etc.

Some ways of eliciting emotions and some induced emotions are listed in Table 1.

\section{Offline or Online Recognition}

In some studies, emotion recognition on the spot is really important such as monitoring patients while taking medicine, so online methods are of importance in those applications. For example, Iacoviello et $\mathrm{al}^{81}$ an effective, general and complete classification method for EEG signals was introduced. In this study, selfinduction was used as emotion elicitation. Wavelet transform (WT), principle component analysis (PCA) and support vector machine (SVM) were used to process and classify EEG signals. Also, Sourina et $\mathrm{al}^{83}$ introduced an online emotion recognition study which used spatial time fractal to characterize brain states. A vital issue in online recognition systems is that processing methods must be fast and precise. Fractal transform is one of these methods that was used in several related studies. Sourina et $\mathrm{al}^{86}$ determined brain responses using fractal transform following stimulation by music. Also they calculated Renyi entropy as well. Liu et $\mathrm{a}^{55}$ calculated Higuchi's fractal dimension. They processed EEG signals while participants were listening to music.

The other type of emotion recognition systems is offline. For example, Zhang and $\mathrm{Li}^{85}$ recognized positive and negative emotions using neuro fuzzy method offline. In this study, an unsupervised clustering method and adaptive neuro fuzzy inference system (ANFIS) were used. Clustering was used in early steps for creating primary information related to emotions. Emotions were elicited by International Affective Picture System (IAPS)

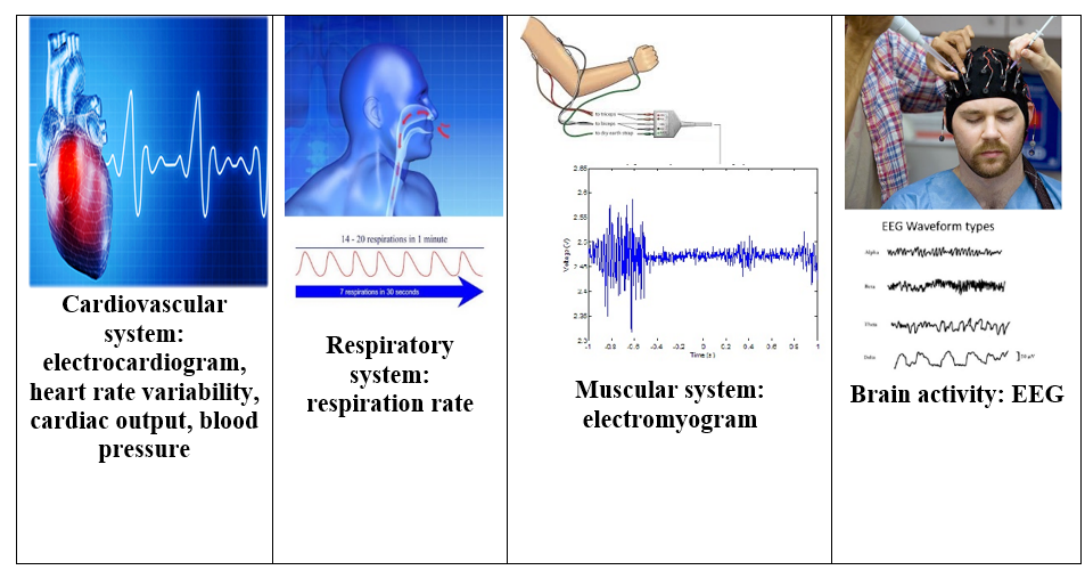

Figure 1. Types of Physiologic Records. 
Table 1. Different Kinds of Emotion Stimulation

\begin{tabular}{lll}
\hline Stimulation & Emotion & Ref. \\
\hline Pictures & Valence-arousal model & 19 \\
& Negative and positive emotions & 54 \\
& Happiness, fear, neutral, sadness & 27 \\
& Valence-arousal model & 17 \\
& Happiness, fear, neutral, sadness, anger & 43 \\
& Negative and positive emotions & 42 \\
& Negative and positive emotions & 97 \\
Video clips & Happiness, sadness, disgust, amusement, fear, & 96 \\
& surprise, anxiety, anger, neutral & 44 \\
& Valence-arousal model & 45 \\
& Happiness, disgust, fear, surprise, anger, neutral & 57 \\
& Valence-arousal model & 58 \\
& Valence-arousal model & 61 \\
& Valence-arousal model & 62 \\
& Happiness, sadness, fear, surprise, anger & 63 \\
\hline
\end{tabular}

images. In this study, EEG signals and visual information were recorded.

\section{Emotion Models}

Another problem in emotion recognition studies is the number of elicited emotions and the emotion model. Some studies, according to discrete model of emotions, consider a specific number of emotions and others according to valence-arousal model suppose more emotions. For example, Murugappan et $\mathrm{al}^{45,48,52}$ studied anger, fear, surprise, happiness emotions according to discrete emotion model, while Koelstra et al, ${ }^{51}$ Koelstra and Patras, ${ }^{53}$ and Hidalgo-Munoz et $\mathrm{al}^{24}$ studied emotions according to the valence-arousal model.

\section{Public Databases}

There are some public emotion databases which can be used by researchers for free. The advantage of public databases is that researchers do not need any laboratory and specific recording systems, appropriate condition, shield environment, etc. Also, they do not need participants and they will have reliable and free databases. In this section, some available databases are described.

\section{DEAP Database}

This multimodal database was recorded by Koelstra et $\mathrm{al}^{51}$ in 2 laboratories (Geneva and Twente) in 2012. In this database, 40 video clips were used to elicit emotions according to valence arousal model. Thirty-two individuals participated and 32-channel EEG signals, 4-channel EMGs, 4 EOG signals, 2-channel GSR signal, 2 ERG signals, temperature in a single channel, single channel respiration rate and 1-channel blood volume pressure were recorded. Five indexes including arousal, valence, like/ dislike, dominance and familiarity were reported by each participant. Raw and preprocessed signals from all participants and also face videos from 22 of them are available in this dataset. More detailed descriptions can be found in https://www.eecs.qmul.ac.uk/mmv/datasets/deap/index. html. Figure 2 shows selected emotional videos in valence arousal plane.

\section{SJTU Emotion EEG Dataset (SEED)}

Zheng and $\mathrm{Lu}^{98}$ recorded SJTU emotion EEG Dataset. This dataset contains EEG signals from 15 individuals while watching Chinese video clips. Figure 3 shows a participant while watching clips. Emotions were considered as positive, negative and natural. Participants filled a questionnaire after watching videos. EEGs were recorded in three sessions to evaluate stability of patterns and neural signatures among participants and sessions, the interval between 2 sessions was one or more weeks. EEG signals were recorded according to the 10-20 international standard system. Raw and preprocessed signals and also face videos are available. For more details refer to http:// bcmi.sjtu.edu.cn/ seed/index.html.

\section{MAHNOB-HCl database}

Soleymani et $\mathrm{al}^{96}$ recorded MAHNOB-HCI database, a multimodal database in 2012. This database included several multimodal signals, 32 channels EEG signals, 3 channels ECG signals, 2 channels ERG, 2 channels GSR, respiration amplitude and skin temperature signals. Multimodal signals were recorded from 27 individuals while watching video clips and pictures. This database included

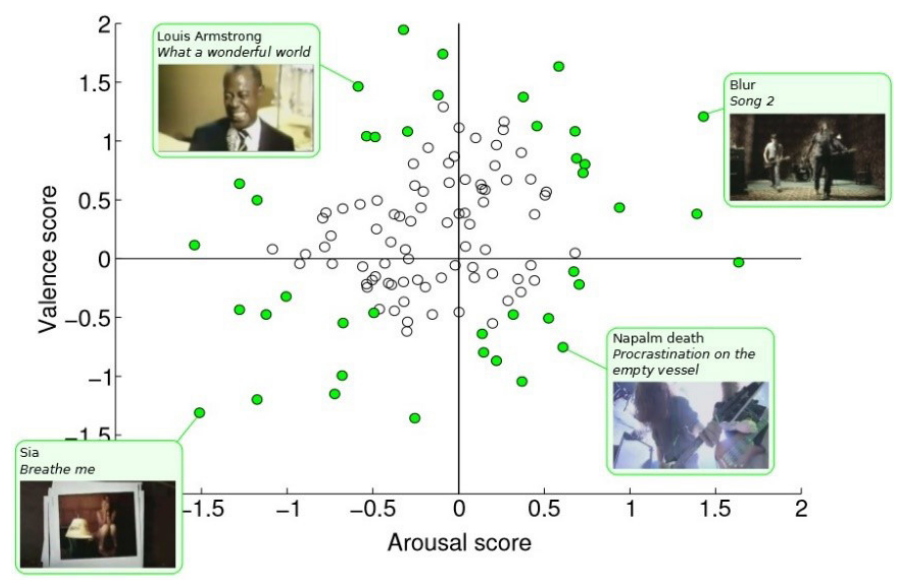

Figure 2. Valence and Arousal Values of Video Clips in DEAP Database. Included video clips are shown in green. ${ }^{51}$ 

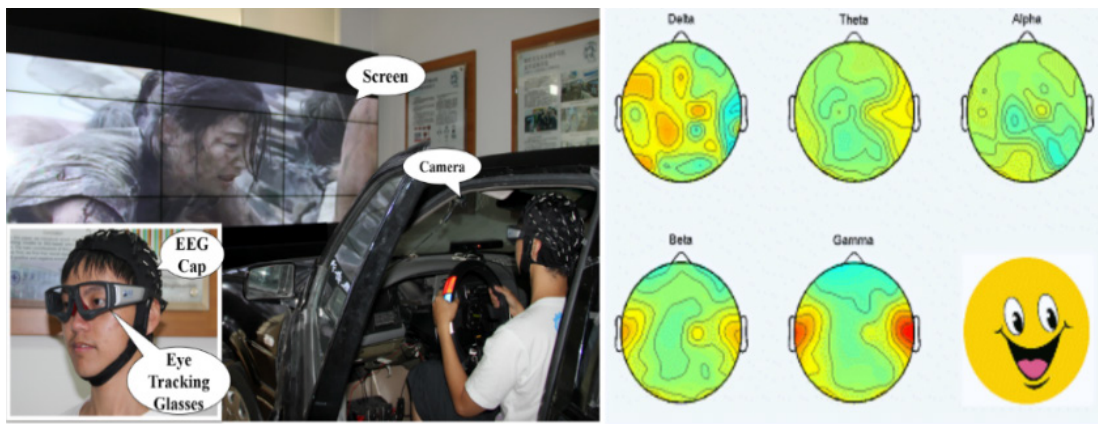

Figure 3. SJTU Emotion EEG Dataset (SEED) setup. ${ }^{98}$

2 separate sessions, in the first session video clips were displayed and participants immediately filled a questionnaire about their feelings after watching clips. In the second session, short videos and pictures were displayed once with right and wrong labels and once without labels. Their comments about their feelings were evaluated. Signals were recorded according to the 10-20 international standard system. For more details about this database refer to https://mahnob-db.eu/hci-tagging/.

These databases have been used in several studies, Table 2 shows a brief description and references which have used biological signals in these databases so far.

\section{Previous Emotion Studies}

Emotion and Normal Cases

In this section we review previous studies which evaluate emotions in normal individuals. Weinreich et $\mathrm{al}^{26}$ measured variations of alpha frequency band in frontal lobe from an oddball paradigm. Participants were asked to describe each image regardless of the emotion of the image. 16-channel EEG signals were recorded from 20 female and 8 male participants.

Hidalgo-Munoz et $\mathrm{al}^{24}$ studied EEG signals of 26 females while watching emotional images from IAPS. This study considered emotions according to the valence-arousal model. In the processing step, they used spectral turbulence (ST), a method which was inspired by ECG studies. Results show that the left temporal lobe has considerable activity during emotion elicitation. Koelstra and Patras ${ }^{53}$ recorded EEG signals from several participants according to the valence-arousal model. They showed video clips in order to evoke emotions. The details are described in section 1.5 and Table 2. In this study, power spectral density of EEG sub-bands was calculated and active units (AU) were detected from face videos of participants. Then a combination of features was applied. Hidden Markov Model (HMM) and GentleBoost were used as the classifiers. Results showed that the combination of face videos and EEG signals improved the accuracy.

Lee et $\mathrm{al}^{54}$ proposed an emotion recognition system based on fuzzy logic. They used video clips to elicit emotions and recorded EEG signals from 12 participants. They extracted dynamic features from emotional states and 3D fuzzy GIST and 3D fuzzy tensor to extract brain features in a semantic level. Independent component analysis (ICA) was used to remove artifacts. ANFIS was used to classify emotions, results showed the performance of the proposed method.

Haung et $\mathrm{al}^{60}$ presented a multimodal approach to recognize emotions. In this study EEG signals from MAHNOB-HCI database were used. Discriminant power spectrum and difference power spectrum were extracted from EEG signals of 27 participants. Local binary patterns (LBP) were extracted from videos of participants' faces. Then fusion in features and decisions were applied. Finally, SVM and KNN were used as classifiers. Results showed that using multimodal data, gives better recognition results.

Bozhkov et $\mathrm{al}^{31}$ considered valence-arousal model for emotions and recorded EEG signals from 26 females viewing IAPS pictures. They used Echo state networks (ESN) to cluster and classify positive and negative emotions. They obtained the desired results and demonstrated the performance of their proposed method.

Mavratzakis et $\mathrm{al}^{36}$ evaluated event related potentials (ERPs) of 27 individuals during watching pictures. In this study, three picture databases were used as stimuli: KDEF (Karolinska Directed Emotional Faces Database), RAFD (Radboud Faces Database) and IAPS. After statistical analysis of ERP components, results showed that emotions did not influence on P1 component. Also, N170 increased during watching emotional pictures but N100 was not sensitive to emotion changes. Moreover, early posterior negativity (EPN) increased during watching fearful images.

\section{Emotions and Neural Disorders}

An interesting part of emotion studies is studies which evaluate psychological diseases and disorders through emotion recognition. In this section, studies about some disorders such as Parkinson's disorder (PD), autism spectrum disorder (ASD), schizophrenia, depression, etc. were reviewed. Yuvaraja et $\mathrm{al}^{88}$ extracted higher order spectral features from EEG signals and evaluated emotion changes between PD patients and normal individuals. EEG signals were recorded from $20 \mathrm{PD}$ patients and 20 normal participants while watching video clips. Samples were classified into six basic emotions (sadness, happiness, fear, anger, surprise and disgust) 


\begin{tabular}{|c|c|c|c|c|c|c|}
\hline Name & Participants & Signals & Stimulation & Emotion & Supplementary Files & Ref. \\
\hline DEAP & 32 & $\begin{array}{l}32 \text { EEGs, } 4 \text { EMGs, } 4 \\
\text { EOGs, } 1 \text { GSR, } 1 \text { RR, } 1 \\
\text { Plethy }^{\mathrm{a}}, 1 \text { Temp }^{\mathrm{b}}\end{array}$ & 40 video clips & $\begin{array}{l}40 \text { emotions according to } \\
\text { valence arousal model }\end{array}$ & Face videos & $44,51,57,58,87$ \\
\hline MAHNOB-HCI & 30 & $\begin{array}{l}16 \text { EEGs, } 3 \text { ECGs, } 2 \\
\text { GSRs, } 1 \text { RR, } 1 \text { Temp }\end{array}$ & $\begin{array}{l}20 \text { Video clips and } \\
\text { pictures }\end{array}$ & $\begin{array}{l}\text { Happiness, Sadness, disgust, } \\
\text { amusement, fear, surprise, } \\
\text { anxiety, anger, neutral }\end{array}$ & $\begin{array}{l}\text { Face videos (522), eye } \\
\text { gaze }\end{array}$ & $53,60,96$ \\
\hline SEED & 15 & 15 EEGs & 15 video clips & $\begin{array}{l}\text { Positive, neutral and } \\
\text { negative emotions }\end{array}$ & Face videos & 59,98 \\
\hline
\end{tabular}

a Plethysmograph, ${ }^{\mathrm{b}}$ Temperature.

through SVM classifier. Results showed PD patients have weaker emotions in comparison with normal individuals, especially for negative emotions. Yeung et a ${ }^{90}$ examined cortical connectivity of autistic children while watching KDEF face pictures and compared them with normal children. EEG signals of 18 autistic children and 18 normal children were recorded during stimuli and then analyzed using theta coherence index (cortical connectivity index). This study showed that autistic children have deficiency in emotion recognition. Also, there was no theta coherence modulation while normal children had theta coherence modulation in the right frontal lobe in response to emotional faces. Theta coherence modulation in response to emotions is related to social deficiency of autistic children.

Schizophrenia can be detected by emotion stimulation. Brennan et $\mathrm{al}^{89}$ examined this hypothesis by processing ERP signals. This study used international BRAINnet database, including 108 schizophrenic patients and 108 normal cases. All individuals watched emotional pictures including sadness, fear, anger, disgust and happiness and simultaneously ERPs were recorded in conscious and non-conscious conditions. Then significant differences among 2 groups were achieved through analysis of variance (ANOVA). Results showed that schizophrenic patients had shorter brain activity, about $70 \mathrm{~ms}$. Also, schizophrenic patients in response to disgust had positive shifts after $70 \mathrm{~ms}$ and normal people had negative shifts in response to fear and anger in comparison with happiness in temporal-occipital regions.

Croft et $\mathrm{al}^{95}$ detected emotion deficiency in Huntington's patients via ERPs. In this study, EEG signals from 11 Huntington's patients and 11 normal individuals were recorded while participants expressed emotions such as scramble, neutral, happiness, anger and disgust. Results showed lower accuracies for negative emotions such as disgust, neutral and anger due to decreased functionality.

Psychogenic non-epileptic seizures (PNES) are unknown among epileptic seizures. Recent studies showed that PNES patients have impairments in control of their emotions. Urbanek et $\mathrm{al}^{94}$ evaluated this hypothesis. In this study, EEG signals from 56 patients and 68 normal individuals during emotion stimulation were recorded. Results demonstrated that these patients have weaker emotions, more negative feelings and stronger control on their emotions than normal people.
Tseng et al evaluated phase synchrony and EEG activation oscillation in Asperger syndrome (AS) patients while they were recognizing emotions from face images. ${ }^{40}$ AS group included 10 individuals and the normal group consisted of 10 individuals. Emotions were stimulated by pictures. Results demonstrated that AS group had no determined N400 in response to pictures, also, they showed lower synchrony in temporal and parietal-occipital lobes at delta/theta and weaker phase synchronization in separate regions of brain.

Akar et al examined brain dynamics of major depressive disorder (MDD) patients during stimulation using positive and negative emotions. ${ }^{72}$ They used music as stimulation. Three different situations including noisy environment, relaxation and listening to music were considered. EEG signals from 15 MDD patients and 15 normal people were recorded and analyzed using nonlinear methods. Some kinds of complexity measures such as Lempel-Ziv, Kolmogorov were calculated and then significant differences were evaluated by ANOVA measure. This study demonstrated that MDD patients have more complex EEG signals in parietal and frontal lobes comparing to normal people. Also EEG signals of these individuals had lower complexity in frontal and parietal lobes while listening to music compared to other situations.

$\mathrm{Li}$ et al evaluated large scale functional brain networks of depressed people and normal ones using graph theory. ${ }^{34}$ Participants' emotions were elicited by Ekman pictures including positive, negative and neutral emotions. Simultaneously, EEG signals were recorded from 16 depressed and 14 normal participants. In this study, EEG signals were processed by extracting coherence in frequency bands such as delta, theta, alpha, beta and gamma. Results showed that for depressed participants total coherence values in gamma band were higher than normal people. Also, total coherence among normal participants for negative emotions was higher in gamma band. Moreover, there were abnormal networks in prefrontal and occipital lobes for depressed participants. Table 3 describes recent studies related to emotion recognition.

\section{Conclusion}

In this paper, we reviewed several emotion recognition studies from EEG signals. First, we stated some emotion 
Table 3. Recent Emotion Recognition and Evaluation Studies From EEGs

\begin{tabular}{|c|c|c|c|c|c|}
\hline Emotions & Stimulation & Recorded signals & Method & Results & Ref \\
\hline Positive and negative valence & Music & EEG signals from 5 women & Correlation dimension, ANOVA & Lower correlation dimension while listening to music & 69 \\
\hline Valence arousal model & Video clips & MAHNOB-HCI & $\begin{array}{l}\text { PSD sub bands (EEGs), AU (face } \\
\text { pictures) detection, fusion in } \\
\text { feature and decision levels, HMM, } \\
\text { GentleBoost }\end{array}$ & Better results using 2 modalities (EEG signals and face pictures) & 53 \\
\hline $\begin{array}{l}\text { rSASM (recalibrated Speech Affective } \\
\text { Space Model), 12-PAC (12-Point Affective } \\
\text { Circumplex) }\end{array}$ & Pictures (RAFD database) & EEG signals from 5 children & $\begin{array}{l}\text { KSDE (Kernel Density Estimation), } \\
\text { MFCC (Mel-Frequency Cepstral } \\
\text { Coefficients), MLP }\end{array}$ & $\begin{array}{l}\text { MFCC-rSASM has lower MSE vs MFCC-12PAC and KSDE-12-PAC } \\
\text { was lower than KSDE-rSASM }\end{array}$ & 27 \\
\hline Happiness, sadness & Self-induction & EEG signals & Signal velocity & Sadness has faster velocity than happiness & 78 \\
\hline Three levels of arousal & $\begin{array}{l}\text { Pictures (IAPS database) and } \\
\text { transcutaneous electric nerve } \\
\text { stimulation (TENS) }\end{array}$ & $\begin{array}{l}\text { EEGs, SC, HR and acoustic } \\
\text { startle amplitude from } 30 \\
\text { individuals }\end{array}$ & Frontal alpha asymmetry & $\begin{array}{l}\text { Relative activity in left frontal lobe plays an important role in emotion } \\
\text { controlling }\end{array}$ & 25 \\
\hline Valence-arousal model & Pictures (IAPS database) & EEGs from 26 females & ST, SVM-RFE & Left temporal lobe activated during emotional stimulation & 24 \\
\hline $\begin{array}{l}\text { Neutral, erotic, aversive, pleasant, } \\
\text { unpleasant }\end{array}$ & Pictures (IAPS database) & EEGs from 73 individuals & Spectral analysis, ANOVA & Increase of upper alpha power in central lobe & 92 \\
\hline Neutral, unpleasant & Pictures (IAPS database) & EEGs from 18 individuals & $\begin{array}{l}\text { Dynamic theta in occipital region, } \\
\text { spectral analysis, ANOVA }\end{array}$ & 2 peaks in early theta power increase & 93 \\
\hline Negative and positive & Video clips & EEGs from 12 individuals & $\begin{array}{l}\text { 3D fuzzy GIST, 3D fuzzy tensor, FCM, } \\
\text { ICA, STFT, ANFIS }\end{array}$ & Effectiveness of 3D fuzzy, derived EEG features and ANFIS & 54 \\
\hline $\begin{array}{l}\text { Very happy, somewhat happy, neutral, } \\
\text { sadness, anger, fear }\end{array}$ & Pictures & EEGs from 29 individuals & $\begin{array}{l}\text { ERP amplitude, delay interval theta } \\
\text { power, ANOVA }\end{array}$ & $\begin{array}{l}\text { Higher N170 component and higher theta power in the delay in } \\
\text { response to negative facial expression }\end{array}$ & 39 \\
\hline Pleasant and unpleasant & $\begin{array}{l}\text { Direct and indirect lightening } \\
\text { environment }\end{array}$ & EEGs from 28 individuals & $\begin{array}{l}\text { Spectral analysis, t-test, Pearson's } \\
\text { correlation coefficient }\end{array}$ & $\begin{array}{l}\text {-Correlation between pleasant score and theta power in F8 channel- } \\
\text { theta frequency band as emotion biomarker in different lightening } \\
\text { conditions }\end{array}$ & 79 \\
\hline Neutral, anger, happiness, disgust & Picture (KDEF database) & $\begin{array}{l}\text { EEGs from } 11 \text { Huntington's } \\
\text { disease patients and } 11 \text { normal } \\
\text { individuals }\end{array}$ & ERP analysis, statistical analysis & $\begin{array}{l}\text { Lower emotion recognition accuracy for HD patients due to } \\
\text { decreased emotional function (neutral, disgust and anger) }\end{array}$ & 95 \\
\hline $\begin{array}{l}\text { Pleasantness, tension, happiness, anger, } \\
\text { fear, energy, sadness, tenderness }\end{array}$ & Music & EEGs from 31 individuals & ICA, connectivity, asymmetry, PCA & Correlation between nervous and evoked emotions & 70 \\
\hline Positive, negative, low level and high level & Video clips & EEGs from 36 individuals & Higuchi fractal dimension, MANOVA & $\begin{array}{l}\text { More complexity for EEGs following (while) high level emotion } \\
\text { stimulation }\end{array}$ & 55 \\
\hline Anger, neutral, happiness & Pictures & EEGs from 31 children & FFT, EEG asymmetry, ANOVA & $\begin{array}{l}\text { Attention decreased in children with right temporal-anterior EEG } \\
\text { asymmetry }\end{array}$ & 38 \\
\hline Negative, positive, neutral & $\begin{array}{l}\text { Pictures (Ekman emotion } \\
\text { database) }\end{array}$ & $\begin{array}{l}\text { EEGs from } 16 \text { depressed patients } \\
\text { and } 14 \text { normal individuals }\end{array}$ & Sub band coherence, graph theory & $\begin{array}{l}\text {-Higher coherence of depressed patients at gamma frequency band } \\
\text {-Higher coherence of normal individual in negative stimulation } \\
\text { compared to positive }\end{array}$ & 34 \\
\hline Neutral, happiness, anger, & $\begin{array}{l}\text { Pictures (Ekman emotion } \\
\text { database) }\end{array}$ & $\begin{array}{l}\text { EEGs from } 10 \text { Asperger's } \\
\text { syndrome (AS) patients and } 10 \\
\text { normal individuals }\end{array}$ & $\begin{array}{l}\text { ERP and ERSP analysis, phase } \\
\text { synchronization, MANOVA }\end{array}$ & $\begin{array}{l}\text { The AS group had no visible N400 component and lower delta/theta } \\
\text { synchronization ( } 350-450 \mathrm{~ms} \text { post-stimulus onset) in the temporal } \\
\text { and occipital-parietal regions }\end{array}$ & 40 \\
\hline
\end{tabular}




\begin{tabular}{|c|c|c|c|}
\hline $\begin{array}{l}\text { Pleasantness, tension, happiness, anger, } \\
\text { fear, energy, sadness, tenderness }\end{array}$ & Music & EEGs from 31 individuals & $\begin{array}{l}\text { Acoustic features (music), frequency } \\
\text { band power and asymmetry (EEGs), } \\
\text { PCA, regression model, correlation }\end{array}$ \\
\hline 4 emotions from valence arousal model & Pictures (IAPS database) & EEGs & $\begin{array}{l}\text { ICA, modified kernel density estimatior } \\
\text { (KDE), artificial neural networks }\end{array}$ \\
\hline Negative and positive & Pictures (IAPS database) & EEGs from 26 women & $\begin{array}{l}\text { Amplitude and latency of ERPs, Neural } \\
\text { networks, logistic regression, naïve } \\
\text { Bayes, linear discriminant analysis }\end{array}$ \\
\hline Negative and positive & Music & $\begin{array}{l}\text { EEGs from } 15 \text { MDD patients and } \\
15 \text { normal individuals }\end{array}$ & $\begin{array}{l}\text { Katz fractal dimension, Higuchi } \\
\text { fractal dimension, Shannon entropy, } \\
\text { Lempel-Ziv complexity, Kolmogorov } \\
\text { complexity (KC), ANOVA }\end{array}$ \\
\hline Valence arousal emotions & Pictures (IAPS database) & $\begin{array}{l}\text { EEGs, HR, SCR, Near-Infrared } \\
\text { Spectroscopy (NIRS) from } 20 \\
\text { subjects }\end{array}$ & $\begin{array}{l}\text { EEG power, correlation analysis, } \\
\text { ANOVA }\end{array}$ \\
\hline Disgust & $\begin{array}{l}\text { Self- induction (remembering } \\
\text { unpleasant smell) }\end{array}$ & EEGs from 10 men & WT, PCA, SVM \\
\hline Unpleasantness & $\begin{array}{l}\text { Self- induction (remembering } \\
\text { unpleasant smell) }\end{array}$ & $\begin{array}{l}\text { EEG from C4, P4, T8 and P8 } \\
\text { from } 28 \text { individuals }\end{array}$ & $\begin{array}{l}\text { STFT (gamma and alpha frequency } \\
\text { bands), similarity measure }\end{array}$ \\
\hline Relaxation & Music (Tanpura drone) & EEGs from 10 individuals & $\begin{array}{l}\text { WT, Empirical Mode Decomposition } \\
\text { (EMD), Multifractal detrended } \\
\text { fluctuation analysis (MFDFA) }\end{array}$ \\
\hline Fear, happiness, neutral & $\begin{array}{l}\text { Pictures (MacBrain Face } \\
\text { Stimulus set) }\end{array}$ & EEGs from 47 individuals & ERP analysis, ANOVA \\
\hline $\begin{array}{l}\text { Happiness, anger, fear, sadness, disgust, } \\
\text { surprise }\end{array}$ & Video clips & $\begin{array}{l}\text { Forehead EEGs, SC, BVP, RR } \\
\text { from } 25 \text { individuals }\end{array}$ & $\begin{array}{l}\text { Adaptive weighted linear model, KNN, } \\
\text { SVM, }\end{array}$ \\
\hline $\begin{array}{l}\text { Very happiness, somewhat happiness, } \\
\text { neutral, sadness, anger, fear }\end{array}$ & $\begin{array}{l}\text { Pictures (MacBrain Face } \\
\text { Stimulus set) }\end{array}$ & $\begin{array}{l}\text { EEGs from } 38 \text { schizophrenic } \\
\text { patients and } 42 \text { normal } \\
\text { individuals }\end{array}$ & ERP analysis, ANOVA \\
\hline $\begin{array}{l}\text { Reappraisal and suppression of negative } \\
\text { emotion }\end{array}$ & Pictures (IAPS database) & EEGs from 102 individuals & $\begin{array}{l}\text { Frontal alpha asymmetry (FAA), } \\
\text { ANOVA }\end{array}$ \\
\hline Valence arousal emotions & Pictures (IAPS database) & EEGs from 28 individuals & WT, ANOVA, \\
\hline Valence arousal emotions & Video clips & $\begin{array}{l}\text { EEGs from } 32 \text { individuals (DEAP } \\
\text { database) }\end{array}$ & $\begin{array}{l}\text { Bispectrum analysis, LS-SVM, ANN } \\
\text { (Linear and RBF kernels) }\end{array}$ \\
\hline Valence arousal emotions & Video clips & DEAP database & $\begin{array}{l}\text { Minimum-Redundancy-Maximum- } \\
\text { Relevance(mRMR), SVM, genetic } \\
\text { algorithm-SVM }\end{array}$ \\
\hline Relaxation & Music & $\begin{array}{l}\text { EEGs from } \mathrm{F} 3 \text { and } \mathrm{F} 4 \text { from } 10 \\
\text { men }\end{array}$ & WT, fractal dimension \\
\hline
\end{tabular}

P300 and P200 from parietal and occipital regions play role in emotion recognition

Significant difference in right and anterior region following emotion stimulation (negative valence vs arousal)

Sensitivity of right hemisphere in gamma frequency band following negative emotion stimulation

Music affect alpha and theta frequency bands

EEG forehead signals are sufficient for emotion recognition

Higher N170 for 2 groups at negative emotions vs neutral but higher theta power in schizophrenic patients in delay interval

Greater relative activity in left frontal as reappraisal negative emotions vs normal view of negative emotions 


\begin{tabular}{|c|c|c|c|c|c|}
\hline Happiness, sadness, anger, love & Music & EEGs from 30 individuals & $\begin{array}{l}\text { Time and spectral features, WT, SVM, } \\
\text { KNN, Multilayer perceptron (MLP), }\end{array}$ & Higher accuracy using fusion of features and MLP classifier & 75 \\
\hline Negative, neutral and positive & Video clips & $\begin{array}{l}\text { EEGs from } 15 \text { subjects (SEED } \\
\text { database) }\end{array}$ & $\begin{array}{l}\text { Domain adaptation, subspace } \\
\text { alignment auto-encoder (SAAE) }\end{array}$ & Effectiveness of SAAE in emotion recognition & 59 \\
\hline Valence arousal emotions & Video clips & $\begin{array}{l}\text { EEGs and face expression from } \\
30 \text { subjects (MAHNOB-HCl } \\
\text { database) }\end{array}$ & $\begin{array}{l}\text { multimodal approach, Spectral power } \\
\text { difference, discriminant spectral power, } \\
\text { KNN, ANOVA, fusion }\end{array}$ & Effectiveness of multimodal approach & 60 \\
\hline Happiness, fear, neutral & Pictures (Ekman database) & $\begin{array}{l}\text { EEG and Transcranial magnetic } \\
\text { stimulation (TMS) from } 12 \\
\text { individuals }\end{array}$ & P1-N1 component, ANOVA & $\begin{array}{l}\text { P1-N1 component reduction in medial prefrontal cortex, first P1-N1 } \\
\text { in right hemisphere and second in left }\end{array}$ & 35 \\
\hline Happiness, fear, neutral & $\begin{array}{l}\text { Pictures (RAFD, KDEF, IAPS, } \\
\text { GAPED' databases) }\end{array}$ & $\begin{array}{l}\text { EEGs and facial } \\
\text { electromyography (fEMG) from } \\
27 \text { individuals }\end{array}$ & ERP, statistical analysis & $\begin{array}{l}\text {-Emotion had no effect on P1 component } \\
\text {-Increase of N170 amplitude with emotion stimulation }\end{array}$ & 36 \\
\hline Happiness, sadness, neutral & Music & EEGs from 19 individuals & $\begin{array}{l}\text { Multi variation autoregressive model, } \\
\text { connectivity, SVM }\end{array}$ & $\begin{array}{l}\text {-Positive correlation between valence and frontal inter-hemispheric } \\
\text { stream } \\
\text {-Negative correlation between bilateral connectivity in parietal lobe }\end{array}$ & 76 \\
\hline Negative & Pictures (IAPS database) & EEGs from 25 individuals & ERP analysis, statistical analysis & $\begin{array}{l}\text { P3 component amplitude was modulated following emotional } \\
\text { stimulation in parietal lobe }\end{array}$ & 32 \\
\hline Valence arousal emotions & Pictures (IAPS database) & EEGs from 26 subjects & $\begin{array}{l}\text { Clustering and classification by Echo } \\
\text { state networks (ESN) }\end{array}$ & Echo state networks were better than classic networks & 31 \\
\hline Valence arousal emotions & Video clips & $\begin{array}{l}\text { EEG signals and peripheral } \\
\text { signals (DEAP database) }\end{array}$ & $\begin{array}{l}\text { Spectral and time features, multiple- } \\
\text { fusion-layer based ensemble classifier } \\
\text { of stacked auto-encoder (MESAE) }\end{array}$ & Preference of MESAE method vs classic methods & 87 \\
\hline Fear, anger & Pictures (POFA²) & $\begin{array}{l}\text { High density EEGs from } 11 \\
\text { young women and } 11 \text { adult } \\
\text { women }\end{array}$ & $\begin{array}{l}\text { ERP analysis ( } N 170 \text { modulation), brain } \\
\text { source localization }\end{array}$ & Change in N170 amplitude, age effects on emotions & 91 \\
\hline Anger, happiness, neutral & $\begin{array}{l}\text { Pictures (Ekman and Friesen's } \\
\text { collection) }\end{array}$ & EEG signals from 46 subjects & $\begin{array}{l}\text { Event-related spectral perturbations, } \\
\text { ANOVA }\end{array}$ & $\begin{array}{l}\text {-Theta synchronization lead to increase in low depression patients } \\
\text { following happiness stimulation } \\
\text {-Increase of theta synchronization due to anger elicitation in high } \\
\text { depression patients }\end{array}$ & 99 \\
\hline $\begin{array}{l}\text { Sad, disgust, fear, anger, happy and } \\
\text { surprise }\end{array}$ & $\begin{array}{l}\text { Pictures (IAPS database), } \\
\text { sounds (IADS }{ }^{3} \text { database), } \\
\text { video clips }\end{array}$ & EEG signals from 57 subjects & $\begin{array}{l}\text { Wavelet packet transform, Hurst } \\
\text { exponent, K-nearest Neighbour (KNN), } \\
\text { Probabilistic Neural Network (PNN) }\end{array}$ & $\begin{array}{l}\text {-Beta as the most discriminative frequency band } \\
\text {-Sad emotion had higher accuracy }(82.32 \%)\end{array}$ & 100 \\
\hline Valence arousal emotions & Video clips & $\begin{array}{l}\text { EEG signals and peripheral } \\
\text { signals (DEAP database) }\end{array}$ & $\begin{array}{l}\text { Reinforcement online learning (ROL), } \\
\text { support vector regression (SVR), least } \\
\text { square regression (LS) }\end{array}$ & $\begin{array}{l}\text { Reduced learning time for Least square reinforcement learning and } \\
\text { support vector reinforcement learning methods }\end{array}$ & 101 \\
\hline Positive and negative & Pictures (GAPED database) & EEG signals from 12 subjects & $\begin{array}{l}\text { Power Spectral Density (PSD), Signal } \\
\text { Power (SP) and Common Spatial } \\
\text { Pattern (CSP), Linear Discrimination } \\
\text { Analysis (LDA) }\end{array}$ & Higher accuracy for finding better electrode arrangement & 102 \\
\hline
\end{tabular}

Geneva affective picture database $=$ GAPED, ${ }^{2}$ Pictures of Facial Affect=POFA, ${ }^{3}$ Inter-national Affective Digitized Sounds $=$ IADS 
approaches and theories. Then we described different components of emotion recognition systems: different kinds of biologic measurements (EEG, ECG, etc) offline vs online recognition systems, different types of emotional stimulation, and the specific emotion models which have been used in studies (valence-arousal model and discrete model). Since EEG has become more and more common in emotion recognition applications in recent years, our main focus was on the subject of emotion recognition through EEG signals. So different papers and studies were reviewed in order to cover this issue. Attempts were also made to support recent, valid and reliable studies for young researchers who are interested in this field.

Conflict of Interest Disclosures

The authors declare that they have no conflict of interests.

Ethical Statement

Not applicable.

\section{Acknowledgment}

We would like to thank Science and Research Branch, Islamic Azad University due to their support.

\section{References}

1. Thagard P. Mind: Introduction to Cognitive Science. Cambridge, MA: MIT press; 2005.

2. Oately K. Best Laid Schemes: The Psychology of Emotions. Cambridge: Cambridge University Press; 1992.

3. Nussbaum MC. Upheavals of Thought. Cambridge: Cambridge University Press; 2001.

4. Ekman P, Friesen WV, O'Sullivan M, Chan A, DiacoyanniTarlatzis I, Heider K, et al. Universals and cultural differences in the judgments of facial expressions of emotion. J Pers Soc Psychol. 1987;53(4):712-7.

5. Kessous L, Castellano G, Caridakis G. Multimodal emotion recognition in speech-based interaction using facial expression, body gesture and acoustic analysis. J Multimodal User Interfaces. 2010;3(1):33-48. doi: 10.1007/s12193-0090025-5.

6. Kaynak O, Alpaydin E, Oja E, Xu L, Raouzaiou A, loannou S, et al. An Intelligent Scheme for Facial Expression Recognition. in Artificial Neural Networks and Neural Information Processing - ICANN/ICONIP. 2003;2714:182-182.

7. Park C, Ryu J, Sohn J, Cho H. An Emotion Expression System for the Emotional Robot. In: IEEE International Symposium on Consumer Electronics, 2007. ISCE 2007; 20-23 June 2007; Irving, TX, USA: IEEE. p.1-6. doi: 10.1109/ISCE.2007.4382195.

8. Rani P, Sarkar N. A New Approach to Implicit Human-Robot Interaction Using Affective Cues. In: Mobile Robots: towards New Applications; 2006. doi: 10.5772/4693.

9. Apolloni B, Howlett R, Jain L, Sim KB, Jang IH, Park CH. The Development of Interactive Feature Selection and GA Feature Selection Method for Emotion Recognition. In KnowledgeBased Intelligent Information and Engineering Systems, Lecture Notes in Computer Science book series (LNCS, volume 4694); 2007. p.73-81.

10. Kim KH, Bang SW, Kim SR. Emotion recognition system using short-term monitoring of physiological signals. Med Biol Eng Comput. 2004;42(3):419-27. doi: 10.1007/bf02344719.

11. Kim J, Andre E. Emotion recognition based on physiological changes in music listening. IEEE Trans Pattern Anal Mach Intell. 2008;30(12):2067-83. doi: 10.1109/tpami.2008.26.

12. Yuen CT, San WS, Seong TC, Rizon M. Classification of Human Emotions from EEG Signals using Statistical Features and Neural
Network. International Journal of Integrated Engineering. 3) 1;2009):71-9.

13. Li M, Lu BL. Emotion classification based on gamma-band EEG. Conf Proc IEEE Eng Med Biol Soc. 2009;2009:1323-6. doi: 10.1109/iembs.2009.5334139.

14. 14. Schaaff K, Schultz T. Towards emotion recognition from electroencephalographic signals. Affective Computing and Intelligent Interaction and Workshops, 2009. ACIl 2009. 3rd International Conference on; 10-12 September 2009. doi: 10.1109/ACII.2009.5349316.

15. Petrantonakis PC, Hadjileontiadis LJ. EEG-Based Emotion Recognition Using Hybrid Filtering and Higher Order Crossings. In: 2009 3rd International Conference on Affective Computing and Intelligent Interaction and Workshops; 10-12 September 2009; Amsterdam. p. 1-6. doi: 10.1109/ACII.2009.5349513.

16. Khalili Z, Moradi MH. Emotion Recognition System Using Brain and Peripheral Signals: Using Correlation Dimension to Improve the Results of EEG. In: International Joint Conference on Neural Networks, IJCNN 2009, Atlanta, Georgia, USA; 14 19 June 2009. doi: 10.1109/IJCNN.2009.5178854.

17. Macas M, Vavrecka M, Gerla V, Lhotska L. Classification of the emotional states based on the EEG signal processing. In: International Conference on Information Technology and Applications in Biomedicine, 2009. ITAB 2009. 9th; 4-7 November 2009. doi: 10.1109/ITAB.2009.5394429.

18. Hosseini SA, Khalilzadeh MA. Emotional stress recognition system using EEG and psychophysiological signals: Using New Labelling Process of EEG Signals in Emotional Stress State. In: Biomedical Engineering and Computer Science (ICBECS) 2010 International Conference on; 23-25 April 2010. doi: 10.1109/ ICBECS.2010.5462520.

19. Petrantonakis PC, Hadjileontiadis LJ. Adaptive extraction of emotion-related EEG segments using multidimensional directed information in time-frequency domain. Conf Proc IEEE Eng Med Biol Soc. 2010;2010:1-4. doi: 10.1109/iembs.2010.5626588.

20. Petrantonakis PC, Hadjileontiadis LJ. Emotion recognition from EEG using higher order crossings. IEEE Trans Inf Technol Biomed. 2010;14(2):186-97. doi: 10.1109/titb.2009.2034649.

21. Petrantonakis PC, Hadjileontiadis LJ. A novel emotion elicitation index using frontal brain asymmetry for enhanced EEG-based emotion recognition. IEEE Trans Inf Technol Biomed. 2011;15(5):737-46. doi: 10.1109/titb.2011.2157933.

22. Hosseini SA, Khalilzadeh MA, Naghibi-Sistani MB, Niazmand V. Higher Order Spectra Analysis of EEG Signals in Emotional Stress States. In: Information Technology and Computer Science (ITCS), 2010 Second International Conference on, Kiev, Ukraine; 24-25 July 2010. doi: 10.1109/ITCS.2010.21.

23. Petrantonakis PC, Hadjileontiadis LJ. An emotion elicitation metric for the valence/arousal and six basic emotions affective models: a comparative study. In: Information Technology and Applications in Biomedicine (ITAB), 2010 10th IEEE International Conference on; Corfu, Greece; 3-5 Nov 2010. doi: 10.1109/ITAB.2010.5687675.

24. Hidalgo-Munoz AR, Lopez MM, Pereira AT, Santos IM, Tome AM. Spectral turbulence measuring as feature extraction method from EEG on affective computing. Biomed Signal Process Control. 2013;8(6):945-50. doi: 10.1016/j.bspc.2013.09.006.

25. Goodman RN, Rietschel JC, Lo LC, Costanzo ME, Hatfield BD. Stress, emotion regulation and cognitive performance: the predictive contributions of trait and state relative frontal EEG alpha asymmetry. Int J Psychophysiol. 2013;87(2):115-23. doi: 10.1016/j.ijpsycho.2012.09.008.

26. Weinreich A, Stephani T, Schubert T. Emotion effects within frontal alpha oscillation in a picture oddball paradigm. Int J Psychophysiol. 2016;110:200-6. doi: 10.1016/j. ijpsycho.2016.07.517.

27. Othman M, Wahab A, Karim I, Dzulkifli MA, Alshaikli IFT. EEG Emotion Recognition Based on the Dimensional Models 
of Emotions. Procedia Soc Behav Sci. 2013;97:30-7. doi: 10.1016/j.sbspro.2013.10.201.

28. Lahane P, Sangaiah AK. An Approach to EEG Based Emotion Recognition and Classification Using Kernel Density Estimation. Procedia Comput Sci. 2015;48:574-81. doi: 10.1016/j.procs.2015.04.138.

29. Bozhkov L, Georgieva P, Santos I, Pereira A, Silva C. EEGbased Subject Independent Affective Computing Models. Procedia Comput Sci. 2015;53:375-82. doi: 10.1016/j. procs.2015.07.314.

30. Balconi M, Grippa E, Vanutelli ME. What hemodynamic (fNIRS), electrophysiological (EEG) and autonomic integrated measures can tell us about emotional processing. Brain Cogn. 2015;95:67-76. doi: 10.1016/j.bandc.2015.02.001.

31. Bozhkov L, Koprinkova-Hristova P, Georgieva P. Reservoir computing for emotion valence discrimination from EEG signals. Neurocomputing. 2017;231:28-40. doi: 10.1016/j. neucom.2016.03.108.

32. Su J, Duan D, Zhang $\mathrm{X}$, Lei $\mathrm{H}$, Wang $\mathrm{C}$, Guo $\mathrm{H}$, et al. The effect of negative emotion on multiple object tracking task: An ERP study. Neurosci Lett. 2017;641:15-20. doi: 10.1016/j. neulet.2017.01.038.

33. Choi D, Sekiya T, Minote N, Watanuki S. Relative left frontal activity in reappraisal and suppression of negative emotion: Evidence from frontal alpha asymmetry (FAA). Int J Psychophysiol. 2016;109:37-44. doi: 10.1016/j. ijpsycho.2016.09.018.

34. Li Y, Cao D, Wei L, Tang Y, Wang J. Abnormal functional connectivity of EEG gamma band in patients with depression during emotional face processing. Clin Neurophysiol. 2015;126(11):2078-89. doi: 10.1016/j.clinph.2014.12.026.

35. Mattavelli G, Rosanova M, Casali AG, Papagno C, Romero Lauro LJ. Timing of emotion representation in right and left occipital region: Evidence from combined TMS-EEG. Brain Cogn. 2016;106:13-22. doi: 10.1016/j.bandc.2016.04.009.

36. Mavratzakis A, Herbert C, Walla P. Emotional facial expressions evoke faster orienting responses, but weaker emotional responses at neural and behavioural levels compared to scenes: A simultaneous EEG and facial EMG study. Neuroimage. 2016;124(Pt A):931-46. doi: 10.1016/j. neuroimage.2015.09.065.

37. Neath-Tavares KN, Itier RJ. Neural processing of fearful and happy facial expressions during emotion-relevant and emotionirrelevant tasks: A fixation-to-feature approach. Biol Psychol. 2016;119:122-40. doi: 10.1016/j.biopsycho.2016.07.013.

38. Solomon B, O'Toole L, Hong M, Dennis TA. Negative affectivity and EEG asymmetry interact to predict emotional interference on attention in early school-aged children. Brain Cogn. 2014;87:173-80. doi: 10.1016/j.bandc.2014.03.014.

39. Brenner CA, Rumak SP, Burns AM, Kieffaber PD. The role of encoding and attention in facial emotion memory: an EEG investigation. Int J Psychophysiol. 2014;93(3):398-410. doi: 10.1016/j.ijpsycho.2014.06.006.

40. Tseng YL, Yang HH, Savostyanov AN, Chien VSC, Liou M. Voluntary attention in Asperger's syndrome: Brain electrical oscillation and phase-synchronization during facial emotion recognition. Res Autism Spectr Disord. 2015;13-14:32-51. doi: 10.1016/j.rasd.2015.01.003

41. Brenner CA, Rumak SP, Burns AMN. Facial emotion memory in schizophrenia: From encoding to maintenance-related EEG. Clin Neurophysiol. 2016;127(2):1366-73. doi: 10.1016/j. clinph.2015.10.061.

42. Balconi M, Brambilla E, Falbo L. BIS/BAS, cortical oscillations and coherence in response to emotional cues. Brain Res Bull. 2009;80(3):151-7. doi: 10.1016/j.brainresbull.2009.07.001.

43. Balconi M, Pozzoli U. Arousal effect on emotional face comprehension: frequency band changes in different time intervals. Physiol Behav. 2009;97(3-4):455-62. doi: 10.1016/j. physbeh.2009.03.023.
44. Zhang $Y$, Ji X, Zhang S. An approach to EEG-based emotion recognition using combined feature extraction method. Neurosci Lett. 2016;633:152-7. doi: 10.1016/j. neulet.2016.09.037.

45. Murugappan M, Nagarajan R, Yaacob S. Combining Spatial Filtering and Wavelet Transform for Classifying Human Emotions Using EEG Signals. J Med Biol Eng. 2011;31(1):4551. doi: 10.5405/jmbe. 710 .

46. Nie D, Wang XW, Shi LC, Lu BL. EEG-based Emotion Recognition during Watching Movies. In: Neural Engineering (NER), 2011 5th International IEEE/EMBS Conference on; Cancun, Mexico; 27 April-1 May 2011. doi: 10.1109/ NER.2011.5910636.

47. Osaka K, Tanioka T, Clocsin R, et al. Electroencephalograph Estimation Method of Measuring Empathic Understanding. In: Natural Language Processing and Knowledge Engineering, 2007. NLP-KE 2007. International Conference on, 30 August-1 September 2007; Beijing: 514-519. doi: 10.1109/ NLPKE.2007.4368080.

48. Murugappan M, Rizon M, Nagarajan R, Yaacob S, Zunaidi I, Hazry D. Lifting scheme for human emotion recognition using EEG. In: Information Technology, 2008. ITSim 2008. International Symposium on; Kuala Lumpur, Malaysia; 26-28 Aug 2008. doi: 10.1109/ITSIM.2008.4631646.

49. Khosrowabadi R, Heijnen M, Wahab A, Quek HC. The Dynamic Emotion Recognition System Based on Functional Connectivity of Brain Regions. In: Intelligent Vehicles Symposium (IV), 2010 IEEE; San Diego, CA, USA; 21-24 June 2010. doi: 10.1109/ IVS.2010.5548102.

50. Nasoz F, Alvarez K, Lisetti CL, Finkelstein N. Emotion recognition from physiological signals using wireless sensors for presence technologies. Cognition Technology Work. 2004;6(1):4-14. doi: 10.1007/s10111-003-0143-x.

51. Koelstra S, Muhl C, Soleymani M, Lee JS, Yazdani A, Ebrahimi T, et al. DEAP: a database for emotion analysis; using physiological signals. IEEE Trans Affect Comput. 2012;3(1):1831. doi: 10.1109/T-AFFC.2011.15.

52. Murugappan $M$, Rizon $M$, Nagarajan R, Yaacob S. EEG feature extraction for classifying emotions using FCM and FKM. Int J Comput Commun Control. 2007;1(2):21-5.

53. Koelstra S, Patras I. Fusion of facial expressions and EEG for implicit affective tagging. Image Vis Comput. 2013;31(2):16474. doi: 10.1016/j.imavis.2012.10.002.

54. Lee G, Kwon M, Kavuri Sri S, Lee M. Emotion recognition based on 3D fuzzy visual and EEG features in movie clips. Neurocomputing. 2014;144:560-8. doi: 10.1016/j. neucom.2014.04.008.

55. Mekler A, Gorbunov I, Gavrilov V. Systemic processes in the brain: The EEG study on the emotions of different hierarchical levels and signs. Int J Psychophysiol. 2014;94(2):191.

56. Khezri M, Firoozabadi M, Sharafat AR. Reliable emotion recognition system based on dynamic adaptive fusion of forehead biopotentials and physiological signals. Comput Methods Programs Biomed. 2015;122(2):149-64. doi: 10.1016/j.cmpb.2015.07.006.

57. Kumar N, Khaund K, Hazarika SM. Bispectral Analysis of EEG for Emotion Recognition. Procedia Comput Sci. 2016;84:31-5. doi: 10.1016/j.procs.2016.04.062.

58. Atkinson J, Campos D. Improving BCl-based emotion recognition by combining EEG feature selection and kernel classifiers. Expert Syst Appl. 2016;47:35-41. doi: 10.1016/j. eswa.2015.10.049.

59. Chai X, Wang Q, Zhao Y, Liu X, Bai O, Li Y. Unsupervised domain adaptation techniques based on auto-encoder for nonstationary EEG-based emotion recognition. Comput Biol Med. 2016;79:205-14. doi: 10.1016/j.compbiomed.2016.10.019.

60. Huang X, Kortelainen J, Zhao G, Li X, Moilanen A, Seppanen $\mathrm{T}$, et al. Multi-modal emotion analysis from facial expressions and electroencephalogram. Comput Vis Image Underst. 
2016;147:114-24. doi: 10.1016/j.cviu.2015.09.015.

61. Lin $\mathrm{YP}$, Wang $\mathrm{CH}, \mathrm{Wu} \mathrm{TL}$, Jeng SK, Chen JH. EEG-Based Emotion Recognition in Music Listening, In: Acoustics, Speech and Signal Processing, 2009. ICASSP 2009. IEEE International Conference on;19-24 April 2009; Taipei, Taiwan; 2009.

62. Khosrowabadi R, Wahab A, Ang KK, Baniasad MH. Affective computation on EEG correlates of emotion from musical and vocal stimuli. Neural Networks, 2009. IJCNN 2009. International Joint Conference on; 14-19 June 2009. doi: 10.1109/IJCNN.2009.5178748.

63. Kuncheva LI, Christy T, Pierce I, Mansoor SP. Multi-modal Biometric Emotion Recognition Using Classifier Ensembles. In International Conference on Industrial, Engineering and Other Applications of Applied Intelligent Systems; Springer, Berlin, Heidelberg; 2011:317-326. doi: 10.1007/978-3-64221822-4_32.

64. Sourina O, Liu Y. A fractal-based algorithm of emotion recognition from EEG using arousal-valence model. Biosignals. Proceedings of the International Conference on Bio-inspired Systems and Signal Processing; 26-29 January 2011; Rome, Italy; 2011.

65. Lin $\mathrm{YP}, \mathrm{Wang} \mathrm{CH}, \mathrm{Wu} \mathrm{TL}$, Jeng $\mathrm{SK}$, Chen JH. EEG-based emotion recognition in music listening: a comparison of schemes for multiclass support vector machine. In: Acoustics, Speech and Signal Processing, 2009. ICASSP 2009. IEEE International Conference on; 19-24 April 2009. doi: 10.1109/ ICASSP.2009.4959627.

66. Liu Y, Sourina O, Nguyen MK. Real-time EEG-based Human Emotion Recognition and Visualization. Cyberworlds (CW); 2010 International Conference on; 20-22 Oct. 2010. doi: 10.1109/CW.2010.37.

67. Lin $\mathrm{YP}$, Wang $\mathrm{CH}, \mathrm{Wu} \mathrm{TY}$, Jeng $\mathrm{SK}$, Chen $\mathrm{JH}$. Multilayer Perceptron for EEG Signal Classification during Listening to Emotional Music. In: TENCON 2007 - 2007 IEEE Region 10 Conference; 30 Oct-2 Nov. 2007. doi: 10.1109/ TENCON.2007.4428831.

68. Lin YP, Wang $\mathrm{CH}, \mathrm{Wu} T \mathrm{TY}$, Jeng SK, Chen JH. Support Vector Machine for EEG Signal Classification during Listening to Emotional Music. In: Multimedia Signal Processing, 2008 IEEE 10th Workshop on, 8-10 Oct; 2008. doi: 10.1109/ MMSP.2008.4665061.

69. Hoseingholizade S, Golpaygani MRH, Monfared AS. Studying emotion through nonlinear processing of EEG. Procedia Soc Behav Sci. 2012;32:163-9. doi: 10.1016/j.sbspro.2012.01.026.

70. Daly I, Malik A, Hwang F, Roesch E, Weaver J, Kirke A, et al. Neural correlates of emotional responses to music: an EEG study. Neurosci Lett. 2014;573:52-7. doi: 10.1016/j. neulet.2014.05.003.

71. Daly I, Williams D, Hallowell J, Hwang F, Kirke A, Malik A, et al. Music-induced emotions can be predicted from a combination of brain activity and acoustic features. Brain Cogn. 2015;101:1-11. doi: 10.1016/j.bandc.2015.08.003.

72. Akdemir Akar S, Kara S, Agambayev S, Bilgic V. Nonlinear analysis of EEGs of patients with major depression during different emotional states. Comput Biol Med. 2015;67:49-60. doi: 10.1016/j.compbiomed.2015.09.019.

73. Maity AK, Pratihar R, Mitra A, Dey S, Agrawal V, Sanyal S, et al. Multifractal Detrended Fluctuation Analysis of alpha and theta EEG rhythms with musical stimuli. Chaos Solitons Fractals. 2015;81:52-67. doi: 10.1016/j.chaos.2015.08.016.

74. Banerjee A, Sanyal S, Patranabis A, Banerjee K, Guhathakurta $T$, Sengupta $R$, et al. Study on brain dynamics by non linear analysis of music induced EEG signals. Physica A: Statistical Mechanics and its Applications. 2016;444:110-20. doi: 10.1016/j.physa.2015.10.030.

75. Bhatti AM, Majid M, Anwar SM, Khan B. Human emotion recognition and analysis in response to audio music using brain signals. Comput Human Behav. 2016;65:267-75. doi: 10.1016/j.chb.2016.08.029.

76. Shahabi H, Moghimi S. Toward automatic detection of brain responses to emotional music through analysis of EEG effective connectivity. Comput Human Behav. 2016;58:231-9. doi: 10.1016/j.chb.2016.01.005.

77. Chanel G, Ansari-Asl K, Pun T. Valence-arousal evaluation using physiological signals in an emotion recall paradigm. In: Systems, Man and Cybernetics, 2007. ISIC. IEEE International Conference on; 7-10 Oct 2007. doi: 10.1109/ ICSMC.2007.4413638.

78. Ahmed MA, Basori AH. The Influence of Beta Signal toward Emotion Classification for Facial Expression Control through EEG Sensors. Procedia Soc Behav Sci. 2013;97:730-6. doi: 10.1016/j.sbspro.2013.10.294.

79. Shin YB, Woo SH, Kim DH, Kim J, Kim JJ, Park JY. The effect on emotions and brain activity by the direct/indirect lighting in the residential environment. Neurosci Lett. 2015;584:28-32. doi: 10.1016/j.neulet.2014.09.046.

80. Placidi G, Avola D, Petracca A, Sgallari F, Spezialetti M. Basis for the implementation of an EEG-based single-trial binary brain computer interface through the disgust produced by remembering unpleasant odors. Neurocomputing. 2015;160:308-18. doi: 10.1016/j.neucom.2015.02.034.

81. lacoviello D, Petracca A, Spezialetti M, Placidi G. A realtime classification algorithm for EEG-based $\mathrm{BCl}$ driven by self-induced emotions. Comput Methods Programs Biomed. 2015;122(3):293-303. doi: 10.1016/j.cmpb.2015.08.011.

82. Chanel G, Rebetez C, Bétrancourt M, Pun T. Emotion Assessment From Physiological Signals for Adaptation of Game Difficulty. IEEE Trans Syst Man Cybern A Syst Hum. 2011;41(6):1052-63. doi: 10.1109/TSMCA.2011.2116000.

83. Sourina $\mathrm{O}$, Wang $\mathrm{Q}$, Liu $\mathrm{Y}, \mathrm{Nguyen} \mathrm{MK}$. A real-time fractal based brain state recognition from EEG and its applications. In: Biosignals; 2011:82-90.

84. Liu Y, Sourina O, Nguyen MK. Real-time EEG-based human emotion recognition and visualization. In: Proceedings of international conference on cyber worlds (CW); 2010:262-9. doi: 10.1109/CW.2010.37.

85. Zhang Q, Lee M. Emotion development system by interacting with human EEG and natural scene understanding. Cogn Syst Res. 2012;14(1):37-49. doi: 10.1016/j.cogsys.2010.12.012.

86. Sourina O, Kulish VV, Sourin A. Novel Tools for Quantification of Brain Responses to Music Stimuli. In: 13th International Conference on Biomedical Engineering. IFMBE Proceedings; 2009

87. Yin Z, Zhao M, Wang Y, Yang J, Zhang J. Recognition of emotions using multimodal physiological signals and an ensemble deep learning model. Comput Methods Programs Biomed. 2017;140:93-110. doi: 10.1016/j.cmpb.2016.12.005.

88. Yuvaraj R, Murugappan M, Mohamed Ibrahim N, Sundaraj $\mathrm{K}$, Omar Ml, Mohamad K, et al. Detection of emotions in Parkinson's disease using higher order spectral features from brain's electrical activity. Biomed Signal Process Control. 2014;14:108-16. doi: 10.1016/j.bspc.2014.07.005.

89. Brennan AM, Harris AW, Williams LM. Neural processing of facial expressions of emotion in first onset psychosis. Psychiatry Res. 2014;219(3):477-85. doi: 10.1016/j. psychres.2014.06.017.

90. Yeung MK, Han YMY, Sze SL, Chan AS. Altered right frontal cortical connectivity during facial emotion recognition in children with autism spectrum disorders. Res Autism Spectr Disord. 2014;8(11):1567-77. doi: 10.1016/j.rasd.2014.08.013.

91. Tsolaki AC, Kosmidou VE, Kompatsiaris IY, Papadaniil C, Hadjileontiadis L, Tsolaki M. Age-induced differences in brain neural activation elicited by visual emotional stimuli: A highdensity EEG study. Neuroscience. 2017;340:268-78. doi: 10.1016/j.neuroscience.2016.10.059

92. Uusberg A, Uibo H, Kreegipuu K, Allik J. EEG alpha and 
cortical inhibition in affective attention. Int J Psychophysiol 2013;89(1):26-36. doi: 10.1016/j.ijpsycho.2013.04.020.

93. Uusberg A, Thiruchselvam R, Gross JJ. Using distraction to regulate emotion: insights from EEG theta dynamics. Int J Psychophysiol. 2014;91(3):254-60. doi: 10.1016/j. ijpsycho.2014.01.006.

94. Urbanek M, Harvey M, McGowan J, Agrawal N. Regulation of emotions in psychogenic nonepileptic seizures. Epilepsy Behav. 2014;37:110-5. doi: 10.1016/j.yebeh.2014.06.004.

95. Croft RJ, McKernan F, Gray M, Churchyard A, GeorgiouKaristianis N. Emotion perception and electrophysiological correlates in Huntington's disease. Clin Neurophysiol. 2014;125(8):1618-25. doi: 10.1016/j.clinph.2013.12.111.

96. Soleymani M, Lichtenauer J, Pun T, Pantic M. A Multimodal Database for Affect Recognition and Implicit Tagging. IEEE Trans Affect Comput. 2012;3(1):42-55. doi: 10.1109/T-AFFC.2011.25.

97. Zhang Q, Lee M. Analysis of positive and negative emotions in natural scene using brain activity and GIST. Neurocomputing.
2009;72(4):1302-6. doi: 10.1016/j.neucom.2008.11.007.

98. Zheng WL, Lu BL. Investigating Critical Frequency Bands and Channels for EEG-based Emotion Recognition with Deep Neural Networks. IEEE Trans Auton Ment Dev. 2015;7(3):162 75. doi: 10.1109/TAMD.2015.2431497.

99. Bocharov AV, Knyazev GG, Savostyanov AN. Depression and implicit emotion processing: An EEG study. Neurophysiol Clin. 2017;47(3):225-30. doi: 10.1016/j.neucli.2017.01.009.

100. Bong SZ, Wan K, Murugappan M, Ibrahim NM, Rajamanickam $\mathrm{Y}$, Mohamad K. Implementation of wavelet packet transform and non linear analysis for emotion classification in stroke patient using brain signals. Biomed Signal Process Control. 2017;36:102-12. doi: 10.1016/j.bspc.2017.03.016.

101. Liu W, Zhang L, Tao D, Cheng J. Reinforcement online learning for emotion prediction by using physiological signals. Pattern Recognit Lett. 2017. doi: 10.1016/j.patrec.2017.06.004.

102. Wei Y, Wu Y, Tudor J. A real-time wearable emotion detection headband based on EEG measurement. Sens Actuators A Phys. 2017;263:614-21. doi: 10.1016/j.sna.2017.07.012. 